\title{
ON THE BURES DISTANCE \\ AND THE UHLMANN TRANSITION PROBABILITY OF STATES ON A VON NEUMANN ALGEBRA
}

\author{
HIDEKI KOSAKI ${ }^{1}$
}

\begin{abstract}
Simple expressions for the Bures distance and the Uhlmann transition probability of states on a von Neumann algebra are obtained. Based on these expressions, certain properties are immediately derived.
\end{abstract}

0. Introduction. For normal states $\varphi, \psi$ on a von Neumann algebra $\mathcal{N}$, the Bures distance $D(\varphi, \psi)$ and the Uhlmann transition probability $P(\varphi, \psi)$ are defined as $\inf || \zeta_{\varphi}-\zeta_{\psi} \|$ and $\sup \left|\left(\zeta_{\varphi} \mid \zeta_{\psi}\right)\right|^{2}$, respectively. Here, the infimum and the supremum are taken over all vectors $\zeta_{\varphi}, \zeta_{\psi}$ satisfying $\varphi=\omega_{\zeta_{\varphi}}, \psi=\omega_{\zeta_{\psi}}$ in any normal representation Hilbert space of $: \pi$. They are related by

$$
D(\varphi, \psi)^{2}=\varphi(1)+\psi(1)-2 P(\varphi, \psi)^{1 / 2}
$$

$($ see $[1,3,8])$.

Since they are regarded as a "distance" and a "transition probability" between two states in quantum mechanics, they have been investigated by theoretical physicists as well as operator algebraists $[\mathbf{1},-\mathbf{4}, \mathbf{8}]$.

The purpose of this article is to obtain certain expressions for $P(\varphi, \psi)$ and $D(\varphi, \psi)$. Our expressions clarify their importance in theoretical physics, and (more importantly) immediately show that the decreasing net $P\left(\left.\varphi\right|_{\Re_{1}},\left.\psi\right|_{\mathscr{R}_{1}}\right)$ (resp. the increasing net $\left.D\left(\left.\varphi\right|^{1} /{ }^{2}, \psi k_{n}^{1}{ }^{2}\right)\right)$ converges to $P(\varphi, \psi(\operatorname{resp} . D(\varphi, \psi)))$ whenever an increasing net $\left\{\mathfrak{K}_{\iota}\right\}_{\iota \in I}$ of von Neumann subalgebras generates $\mathfrak{N}$.

1. Main results. As in $\S 0$, we fix a von Neumann algebra $\mathfrak{R}$ and $\varphi, \psi \in \mathfrak{R}_{*}^{+}$ throughout. We now state our main theorem (which will be proved in \$2) and derive some consequences. However, usually results for only $P(\varphi, \psi)$ are stated from which the corresponding results for $D(\varphi, \psi)$ can be obtained through (1).

THEOREM 1. Let $\left\{\mathfrak{T}_{\iota}\right\}_{\iota \in I}$ be an increasing net of von Neumann subalgebras satisfying $\left(\cup_{\iota \in I} \mathfrak{M}_{\iota}\right)^{\prime \prime}=\mathfrak{N}$. We then have

$$
P(\varphi, \psi)^{1 / 2}=\inf \left\{\sum_{k=1}^{N} \varphi\left(p_{k}\right)^{1 / 2} \psi\left(p_{k}\right)^{1 / 2}\right\} .
$$

Received by the editors November 15, 1982 and, in revised form, January 12, 1983.

1980 Mathematics Subject Classification. Primary 46L 10.

${ }^{1}$ Supported in part by NSF(MCS-8102158). 
Here the infimum is taken over all families $P=\left\{p_{1}, \ldots, p_{N}\right\}$ consisting of finitely many projections such that (i) $p_{i} \perp p_{j}(i \neq j)$, (ii) $\sum_{k=1}^{N} p_{k}=1$, (iii) $p_{1}, \ldots, p_{N} \in \mathfrak{M} \iota_{\iota_{0}}$ for some $\iota_{0} \in I$ (depending upon $P$ ).

Notice that, if $\Re$ is a von Neumann subalgebra, we have $P(\varphi, \psi) \leqslant P\left(\left.\varphi\right|_{\Re},\left.\psi\right|_{\Re}\right)$ from the definition. Also, when $\mathcal{X}$ is the finite-dimensional abelian von Neumann subalgebra generated by projections $p_{1}, \ldots, p_{N}, p_{i} \perp p_{j}(i \neq j), \quad \sum_{k=1}^{N} p_{k}=1$, $P\left(\left.\varphi\right|_{\Re},\left.\psi\right|_{\mathscr{X}}\right)^{1 / 2}$ is exactly $\sum_{k=1}^{N} \varphi\left(p_{k}\right)^{1 / 2} \psi\left(p_{k}\right)^{1 / 2}$.

When $\Re_{\iota}=\Re$ in Theorem 1 , we have

Corollary 2. For $\varphi, \psi \in \mathfrak{R}_{*}^{+}$, we have $P(\varphi, \psi)=\inf \left\{P\left(\left.\varphi\right|_{\Re},\left.\psi\right|_{\Re}\right)\right.$ : $\Re$ is a finite-dimensional abelian von Neumann subalgebra\}.

This is actually a "discrete" version of [2] and closely related to [7]. Noticing condition (iii) in Theorem 1, we immediately have

COROLlaRY 3. If an increasing net $\left\{\mathfrak{R}_{\iota}\right\}_{\iota \in I}$ of von Neumann subalgebras generates $\Re$, then the (decreasing) net $\left\{P\left(\left.\varphi\right|_{\pi_{i}},\left.\psi\right|_{\mathscr{N}_{\imath}}\right)\right\}$ (bounded by $P(\varphi, \psi)$ from below) converges to $P(\varphi, \psi)$.

The following two remarks are in order:

(i) Corollary 2 and (1) mean that

$$
D(\varphi, \psi)=\sup \left\{\left[\sum_{k=1}^{N}\left(\varphi\left(p_{k}\right)^{1 / 2}-\psi\left(p_{k}\right)^{1 / 2}\right)^{2}\right]^{1 / 2}\right\},
$$

where the supremum is taken over all families $P=\left(p_{1}, \ldots, p_{N}\right)$ consisting of finitely many projections in $\Re$ such that $p_{i} \perp p_{j}(i \neq j)$ and $\Sigma_{k=1}^{N} p_{k}=1$. When a physical system is described by $\mathfrak{R}$, each selfadjoint $x=\int_{-\infty}^{\infty} \lambda d e(\lambda)$ in $\mathfrak{R}$ is considered as an observable. Then, for a state $\varphi \in \mathfrak{N}_{*}^{+}$and a partition $\mathbf{R}=\cup_{k=1}^{N} E_{k}$ (of $\mathbf{R}$ into disjoint Borel subsets), $\varphi\left(p_{k}\right)=\int_{E_{k}} d_{\varphi}(e(\lambda))$ (with $p_{k}=\int_{E_{k}} d e(\lambda)$ ) is interpreted as the probability that a measurement of $x$ performed on the system in the state $\varphi$ yields a result lying in $E_{k}$. Thus, $D(\varphi, \psi) \leqslant \varepsilon$ for a small $\varepsilon>0$ means that two states $\varphi, \psi$ give almost similar measurements for any observable $x$ in the sense that $\sum_{k=1}^{N}\left(\varphi\left(p_{k}\right)^{1 / 2}-\psi\left(p_{k}\right)^{1 / 2}\right)^{2} \leqslant \varepsilon^{2}$ (for any partition $\mathbf{R}=\cup_{k=1}^{N} E_{k}$ ). Therefore, the Bures distance is quite suitable to describe a distance between two (physical) states.

(ii) When $\mathfrak{A}$ is the UHF algebra defined by an increasing sequence of finitedimensional factors $\mathfrak{A}_{n}, n=1,2, \ldots$, for two states $\varphi, \psi \in \mathfrak{U}_{+}^{*}, \lim _{n \rightarrow \infty} P\left(\left.\varphi\right|_{\mathfrak{A}_{n}},\left.\psi\right|_{\mathfrak{A}_{n}}\right)$ is exactly the Uhlmann transition probability between the normal extensions $\tilde{\varphi}$, $\tilde{\psi} \in\left(\mathfrak{A}^{* *}\right)_{*}^{+}$on the universal enveloping von Neumann algebra $\mathfrak{A}^{* *}[6]$. Notice that $\lim _{n \rightarrow \infty} P\left(\left.\varphi\right|_{\mathfrak{A}_{n}},\left.\psi\right|_{\mathfrak{A}_{n}}\right)$ can be computed at the matrix algebra level. (This is especially easy when each $\mathfrak{A}_{n}$ is a tensor product, and $\varphi, \psi$ are product states.)

2. Proof of Theorem 1. Obviously the right-hand side of (2) is jointly monotone and upper semicontinuous in $(\varphi, \psi) \in \mathfrak{N}_{*}^{+} \times \mathfrak{R}_{*}^{+}$, while the map $(\varphi, \psi) \mapsto$ $P(\varphi, \psi)^{1 / 2}$ is known to be jointly monotone and continuous. Hence, we may and do assume that $\varphi \leqslant l \psi$ for some $l>0$ (by approximating $\psi$ by $\psi+n^{-1} \varphi, n=1,2, \ldots$, from above). Then we may also assume $\|\psi\| \leqslant 1$ and $\psi$ is faithful. Using the GNS 
construction, we can now assume that $\mathcal{N}$ acts on a Hilbert space $\mathscr{K}$ and $\psi=\omega_{\xi_{0}}$ with some vector $\xi_{0} \in \mathcal{H}\left(\left\|\xi_{0}\right\|=\psi(1)^{1 / 2} \leqslant 1\right)$.

Due to $\varphi \leqslant l \psi$, there exists a positive operator $h \in \mathcal{N}$ such that $\varphi=\omega_{\left.h \xi_{0}\right)}$, $\|h\| \leqslant l^{1 / 2}$ (Sakai's Radon-Nikodym derivative [5]). It is known that $P(\varphi, \psi)^{1 / 2}=$ $\left(h \xi_{0} \mid \xi_{0}\right)$. Thus (due to the remark before Corollary 2) it suffices to show that

$$
\left(h \xi_{0} \mid \xi_{0}\right) \geqslant \inf \left\{\sum_{k=1}^{n} \varphi\left(p_{k}\right)^{1 / 2} \psi\left(p_{k}\right)^{1 / 2}\right\}
$$

(under the above hypotheses).

We choose and fix a small $\varepsilon>0$. Kaplansky's density theorem [5] guarantees the existence of a positive $\tilde{k}$ in $\cup_{\iota \in I}: \Re_{\imath}$ (hence $\tilde{k} \in \mathbb{M}_{\iota_{0}}$ for some $\iota_{0} \in I$ ) satisfying $\left\|(h-\tilde{k}) \xi_{0}\right\| \leqslant \varepsilon$ and $\|\tilde{k}\| \leqslant\|h\| \leqslant l^{1 / 2}$. Introducing the invertible positive operator $k=\tilde{k}+\operatorname{Min}\left(\varepsilon, l^{1 / 2}\right) l \in: \mathbb{R}_{i_{0}}^{+}$, we observe that

$$
\left\|(h-k) \xi_{0}\right\| \leqslant 2 \varepsilon, \quad\|k\| \leqslant 2 l^{1 / 2} .
$$

The obvious estimate $\left|\left((h-k) \xi_{0} \mid \xi_{0}\right)\right| \leqslant 2 \varepsilon$ implies

$$
\left(h \xi_{0} \mid \xi_{0}\right)+2 \varepsilon \geqslant\left(k \xi_{0} \mid \xi_{0}\right) .
$$

Let $k=\int_{0}^{\|k\|} \lambda d e(\lambda)$ be the spectral decomposition so $\left(k \xi_{0} \mid \xi_{0}\right)=\int_{0}^{\|k\|} \lambda d \psi(e(\lambda))$. (Notice that $e(0)=$ strong-lim $\operatorname{li\searrow 0}_{\lambda} e(\lambda)=0$.) For each $n \in \mathbf{N}_{+}$(independent of $\varepsilon$ ), we set

$$
e_{i, n}=e\left(i\|k\| n^{-1}\right)-e\left((i-1)\|k\| n^{-1}\right), \quad i=1,2, \ldots, n,
$$

so $e_{i, n} \perp e_{j, n}(i \neq j), \sum_{i=1}^{n} e_{i, n}=1$, and they are projections in $\mathcal{M}_{\left.\iota_{0}\right)^{\circ}}$ Due to the estimates

$$
\begin{aligned}
\sum_{i=1}^{n} i\|k\| n^{-1} \psi\left(e_{i, n}\right)- & \int_{0}^{\|k\|} \lambda d \psi(e(\lambda)) \leqslant\|k\| n^{-1} \int_{0}^{\|k\|} d \psi(e(\lambda)) \\
& =\|k\| n^{-1} \psi(1) \leqslant 2 l^{1 / 2} n^{-1} \quad(\text { recall }(3) \text { and } \psi(1) \leqslant 1),
\end{aligned}
$$

(4) implies

$$
\left(h \xi_{0} \mid \xi_{0}\right)+2 \varepsilon+2 l^{1 / 2} n^{-1} \geqslant \sum_{i=1}^{n} i\|k\| n^{-1} \psi\left(e_{i, n}\right) .
$$

We now set $\tilde{\psi}=\omega_{k \xi_{0}} \in \Omega_{*}^{+}$and observe that

$$
\tilde{\psi}(e(\mu))=\psi(k e(\mu) k)=\psi\left(\int_{0}^{\mu} \lambda^{2} d e(\lambda)\right)=\int_{0}^{\mu} \lambda^{2} d \psi(e(\lambda)) .
$$

Since $\lambda^{2}$ is increasing, we have

$$
\tilde{\psi}\left(e_{i, n}\right) \leqslant\left\{i\|k\| n^{-1}\right\}^{2} \psi\left(e_{i, n}\right)
$$

Taking the square roots, multiplying by $\psi\left(e_{i, n}\right)^{1 / 2}$, and summing over $i$, we get

$$
\sum_{i=1}^{n} \tilde{\psi}\left(e_{i, n}\right)^{1 / 2} \psi\left(e_{i, n}\right)^{1 / 2} \leqslant \sum_{i=1}^{n} i\|k\| n^{-1} \psi\left(e_{i, n}\right) .
$$


Hence (5) yields

$$
\left(h \xi_{0} \mid \xi_{0}\right)+2 \varepsilon+2 l^{1 / 2} n^{-1} \geqslant \sum_{i=n}^{n} \tilde{\psi}\left(e_{i, n}\right)^{1 / 2} \psi\left(e_{i, n}\right)^{1 / 2} .
$$

We now notice that, for $\alpha, \beta \geqslant 0$, we have $\left(\alpha^{1 / 2}-\beta^{1 / 2}\right)^{2} \leqslant|\alpha-\beta|$. This fact and the Cauchy-Schwarz inequality imply that

$$
\begin{aligned}
\delta & =\left|\sum_{i=1}^{n} \tilde{\psi}\left(e_{i, n}\right)^{1 / 2} \psi\left(e_{i, n}\right)^{1 / 2}-\sum_{i=1}^{n} \varphi\left(e_{i, n}\right)^{1 / 2} \psi\left(e_{i, n}\right)^{1 / 2}\right|^{2} \\
& \leqslant\left[\sum_{i=1}^{n} \psi\left(e_{i, n}\right)\right]\left[\sum_{i=1}^{n}\left(\tilde{\psi}\left(e_{i, n}\right)^{1 / 2}-\varphi\left(e_{i, n}\right)^{1 / 2}\right)^{2}\right] \\
& \leqslant \psi(1)\left[\sum_{i=1}^{n}\left|\tilde{\psi}\left(e_{i, n}\right)-\varphi\left(e_{i, n}\right)\right|\right] \leqslant \sum_{i=1}^{n}\left|(\tilde{\psi}-\varphi)\left(e_{i, n}\right)\right| .
\end{aligned}
$$

Since $\tilde{\psi}-\varphi \in \mathbb{N}_{*}$ is selfadjoint, the positive part $|\tilde{\psi}-\varphi|$ of its polar decomposition [5] enjoys $|(\tilde{\psi}-\varphi)(x)| \leqslant|\tilde{\psi}-\varphi|(x)$ for any $x \in \Re_{+}$. We thus further estimate

$$
\begin{aligned}
\delta & \leqslant \sum_{i=1}^{n}|\tilde{\psi}-\varphi|\left(e_{i, n}\right)=|\tilde{\psi}-\varphi|(1)=\|\tilde{\psi}-\varphi\|=\left\|\omega_{k \xi_{0}}-\omega_{h \xi_{0}}\right\| \\
& \leqslant\left\|(k-h) \xi_{0}\right\|\{\|k\|+\|h\|\}\left\|\xi_{0}\right\| \leqslant 2 \varepsilon\left\{2 l^{1 / 2}+l^{1 / 2}\right\}=6 \varepsilon l^{1 / 2}
\end{aligned}
$$

due to (3).

Therefore, (6) implies

$$
\left(h \xi_{0} \mid \xi_{0}\right)+2 \varepsilon+2 l^{1 / 2} n^{-1}+6^{1 / 2} \varepsilon^{1 / 2} l^{1 / 4} \geqslant \sum_{i=1}^{n} \varphi\left(e_{i, n}\right)^{1 / 2} \psi\left(e_{i, n}\right)^{1 / 2} .
$$

Since $\varepsilon>0$ is arbitrary and $n \in \mathbf{N}_{+}$is independent of $\varepsilon$, this estimate means (2)'. Q.E.D.

\section{REFERENCES}

1. H. Araki, Positive cones, Radon-Nikodym theorems, relative Hamiltonian and Gibbs condition in statistical mechanics ( $C^{*}$-Algebras and their Applications to Statistical Mechanics and Quantum Field Theory (D. Kastler, ed.), North-Holland, Amsterdam, 1976.

2. H. Araki and G. Raggio, A remark on transition probability, Lett. Math. Phys. 6 (1982), 237-240.

3. D. Bures, An extension of Kakutani's theorem on infinite product measures to the tensor product of semifinite von Neumann algebras, Trans. Amer. Math. Soc. 135 (1969), 199-212.

4. S. Gudder, J. P. Marchand and W. Wyss, Bures distance and relative entropy, J. Math. Phys. 20 (1979), 1963-1966.

5. J. Dixmier, Les algèbres d'opérateurs dans l'espace Hilbertien, Gauthier-Villars, Paris, 1969.

6. , Les $C^{*}$-algèbres et leurs représentations, Gauthier-Villars, Paris, 1969.

7. N. Hadjisavvas, On Catoni's generalized transition probability, Comm. Math. Phys. 83 (1982), 43-48.

8. A. Uhlmann, The "transition probability" in the state space of $a{ }^{*}$-algebra, Rep. Math. Phys. 9 (1976), 273-279.

\section{Department of Mathematics, Purdue University, West Lafayette, Indiana 47907}

Current address: Department of Mathematics, University of Pennsylvania, Philadelphia, Pennsylvania 19104-3859 